\title{
Development of a reverse supply chain model for electronic waste incorporating transportation risk
}

\author{
Doan Thi Truc Linh ${ }^{1,2, *}$, Yousef Amer ${ }^{1}$, Sang- Heon Lee ${ }^{1}$, and han Nguyen Ky \\ Phuc ${ }^{3}$ \\ ${ }^{1}$ School of Engineering, University of South Australia, Australia \\ ${ }^{2}$ Department of Industrial Management, Can Tho University, Vietnam \\ ${ }^{3}$ Department of Industrial System Engineering, Ho Chi Minh International University, \\ Vietnam \\ *doalt002@mymail.unisa.edu.au
}

\begin{abstract}
The quantity of Electronic waste (E-waste) is considerably growing due to the rapid development of technology. To diminish the influences of E-waste to the environment and recover raw materials, the reverse supply chain (RSC) has been examined. Most research focuses on minimizing the total cost of the system, however, does not integrate risk factors related to RSC operation. Risks generally derive from transportation activity in E-waste RSC such as delays for pick up, breakdown of trucks, the uncertainty of dangerous materials which might lead to disruptions and higher cost. Therefore, this paper aims to develop a mathematical model for minimizing the total cost of E-waste RSC which integrates transportation risk. A mixed integer linear programming is utilized in the model and addressed by an optimization software. The results of the proposed model can determine the optimal locations and the amount of used products transported within the RSC network. The numerical example also demonstrates that the movement of materials or components in the RSC network is considerably affected by considering transportation risk. The suggested model can assist decision makers about establishing RSC network in which risk elements are incorporated.
\end{abstract}

Keywords: Electronic waste, mixed integer linear programming, supply chain management, transportation risk

\section{Introduction}

E-waste is becoming the largest waste with the generation of 20-50 million tons, and increasing rate from $3 \%$ to $5 \%$ every year (1). To address the E-waste issue, the reverse supply chain (RSC) is being investigated because it can help companies to maintain profits and sustainable development (2).

The cost for RSC system is relatively high since this system may require an enormous initial investment (3). To minimize the total cost of an RSC system, studies have focused on RSC optimization (4-6). Mixed integer linear programming (MILP) formulation is commonly adopted in the literature of RSC modeling (7). Fleichmann et al. (8) introduced a basic RSC network design in the product recovery context. The developed formulation was a multi-echelon, single-product, uncapacitated facility location model for designing RSC, considering cost minimization as the objective function. To evaluate the 
developed model, they applied it in two examples: copier remanufacturing and paper recycling. John and Sridharan (9) presented a mathematical model for multi-layer RSC. This model used MILP and solved by Lingo software. Sensitivity analysis was obtained, and they recommended that the variation of input values can assist managers to have a better decision. In supply chain operations, risk is considered as a potential fluctuation from the initial objective, which can cause non- value added activities at various steps (10). Kumar et al (11) suggested a mathematical model to minimize the total cost with incorporating the different types of risks and their influences on the supply chain system. A study on supply chain risk management was conducted by Thun and Hoeing (12) through investigation of 67 manufacturing companies in German. The research analysed the key risks in supply chain system and examined their probability of occurrence and their impact. The result illustrates that most of supply chain risks derive from inside supply chain which managers can mitigate these risk directly.

In summary, most studies mainly consider investment, processing, disposal and transportation costs in the overall cost of RSC operation. According to Dat et al. (6), transportation cost constitutes a large percentage of total cost for RL. To our best knowledge, transportation risk related to RSC operation is not investigated by most authors. Risks can typically result in shipping of toxic materials contained in E-waste which can affect transportation and the total costs (13). Therefore, the existing models are insufficient for a representative of E-waste RSC model. Hence, this paper aims to develop a multiproduct, multi-echelon RSC model for E-waste to minimize the total cost considering transportation risk.

The remaining sections of this paper are organized as follow: Section 2 contains problem descriptions while Section 3 discusses the modeling of E-waste RSC. The numerical example is presented in Section 4. Lastly, conclusions and future work are discussed in Section 5.

\section{Problem descriptions}

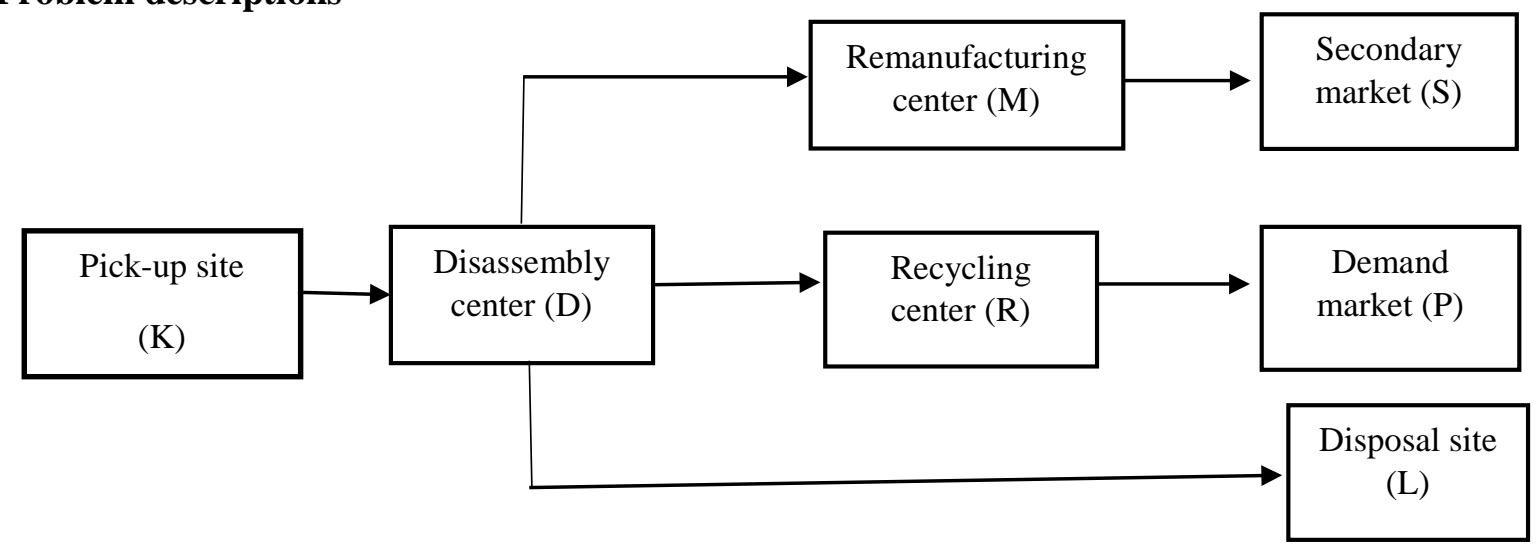

Figure 1. The E-waste reverse supply chain network.

In the RSC model for E-waste (shown in Figure 1), used products from retailers or consumers are first collected at pick-up sites. After that, they are delivered to disassembly centers where these used products are disassembled into different parts or materials at this stage. In the next step, some recyclable materials like metals, plastics are sent to recycling facilities while broken or damaged items are delivered to remanufacturing facilities. Toxic materials or waste will be transported to disposal site for special treatment. Finally, recyclable and renewable materials are transferred to demand and secondary markets respectively. The assumptions of the suggested model are presented as following:

- The sites of pick-up, secondary and demand markets are selected in advance.

- The treatment facilities are limited size

- The unit transportation cost is calculated depending on distance traveled.

- The probability of accident happened and the consequence of the accident are estimated 


\section{Model development}

In the proposed model, the main objective is to minimize total cost (1), involving the sum of constructing cost, operation cost, transportation cost, disposal cost, and risk costs and minus income receiving from selling useful materials. Comparing to existing models, risks associated with transportation activities in the RSC operation are considered in this paper. These risks such as transportation delays, the breakdown of trucks or the uncertainty of hazardous materials normally stem from delivering components or materials between nodes in the RSC network, which can lead to higher transportation cost (14). In this paper, the risk cost, especially transportation risk, can be calculated by multiplying the percentage value of risk score and corresponding transportation cost. Total cost (TC) of the proposed model can be calculated as Equation (1). Constraint (2) indicates all used products are received at collecting areas. The outcomes of disassembly facilities are described by constraints (3)-(5). Constraints (6)-(7) ensure the flow equivalence of different kinds at facilities. Constraints (8)-(11) require that a number of items at disassembly, remanufacturing, recycling facilities and landfill sites do not greater than the maximum capacity of these facilities. Constraints (12)-(13) make sure that the quantities of reusable and recycling materials do not exceed the maximum need of demand and secondary markets. Constraints (14)-(15) show the binary and integer variables.

$$
\begin{aligned}
& T C=\sum_{d=1}^{D} F d_{d} B C_{d}+\sum_{m=1}^{M} F m_{m} B C_{m}+\sum_{r=1}^{R} F r_{r} B C_{r}+\sum_{u=1}^{U} \sum_{k=1}^{K} \sum_{d=1}^{D} Q 1_{u k d} \times O u_{u d}+\sum_{e=1}^{E} \sum_{d=1}^{D} \sum_{m=1}^{M} Q 2_{e d m} \times O e_{e m} \\
& +\sum_{y=1}^{T} \sum_{d=1}^{D} \sum_{r=1}^{R} Q 3_{y d r} \times O y_{y r}+\sum_{u=1}^{U} \sum_{k=1}^{K} \sum_{d=1}^{D} Q 1_{u k d} \times T D_{k m} \times T u_{u}+\sum_{e=1}^{E} \sum_{d=1}^{D} \sum_{m=1}^{M} Q 2_{e d m} \times T D_{d m} \times T e_{e}+\sum_{y=1}^{Y} \sum_{d=1}^{D} \sum_{r=1}^{R} Q 3_{y d r} \times T D_{d r} \times T y_{y} \\
& +\sum_{t=1}^{T} \sum_{d=1}^{D} \sum_{l=1}^{L} Q 4_{t d l} \times T D_{d l} \times T t_{t}+\sum_{e=1}^{E} \sum_{m=1}^{M} \sum_{s=1}^{S} Q 5_{e m s} \times T D_{m s} \times T E_{e}+\sum_{y=1}^{T} \sum_{r=1}^{R} \sum_{p=1}^{P} Q 6_{y r p} \times T D_{r p} \times T Y_{i y}+\sum_{t=1}^{T} \sum_{d=1}^{D} \sum_{l=1}^{L} Q 6_{t d l} \times L C_{t l} \\
& +\sum_{u=1}^{L} \sum_{k=1}^{K} \sum_{d=1}^{D} Q 1_{u k d} \times T D_{k m} \times T u_{u} \times \frac{P O 1_{k d} \times C O 1_{k d}}{M a x\left(P O 1_{k d} \times C O 1_{k d}\right)}+\sum_{e=1}^{D} \sum_{d=1}^{D} \sum_{m=1}^{M} Q 2_{e d m} \times T D_{d m} \times T e_{e} \times \frac{P O 2_{d m} \times C O 2_{d m}}{M a x\left(P O 2_{d m} \times C O 2_{d m}\right)} \\
& +\sum_{y=1}^{T} \sum_{d=1}^{D} \sum_{r=1}^{R} Q 3_{y d r} \times T D_{d r} \times T y_{y} \times \frac{P O 3_{d r} \times C O 3_{d r}}{M a x\left(P O 3_{d r} \times C O 3_{d r}\right)}+\sum_{t=1}^{T} \sum_{d=1}^{D} \sum_{l=1}^{L} Q 4_{t d l} \times T D_{d l} \times T t_{t} \times \frac{P O 4_{d l} \times C O 4_{d l}}{\operatorname{Max}\left(P O 4_{d l} \times C O 4_{d l}\right)} \\
& +\sum_{e=1}^{E} \sum_{m=1}^{M} \sum_{s=1}^{S} Q 5_{e m s} \times T D_{m s} \times T E_{e} \times \frac{P O 5_{m s} \times C O 5_{m s}}{M a x\left(P O 5_{m s} \times C O 5_{m s}\right)}+\sum_{y=1}^{T} \sum_{r=1}^{R} \sum_{p=1}^{P} Q 6_{y r p} \times T D_{r p} \times T Y_{i y} \times \frac{P O 6_{r p} \times C O 6_{r p}}{\operatorname{Max}\left(P O 6_{r p} \times C O 6_{r p}\right)} \\
& -\sum_{e=1}^{E} \sum_{m=1}^{M} \sum_{s=1}^{S} Q 5_{e m s} \times \operatorname{Re}_{e s}-\sum_{y=1}^{Y} \sum_{r=1}^{R} \sum_{p=1}^{P} Q 6_{y r p} \times R y_{y p}
\end{aligned}
$$

Subject to:

$$
\begin{aligned}
& \sum_{d=1}^{D} Q 1_{u k d}=N_{u k}, \forall u, k \\
& \sum_{m=1}^{M} Q 2_{e d m}=\sum_{u=1}^{U}\left(\alpha 1_{e u} \times \sum_{k=1}^{K} Q 1_{u k d}\right), \quad \forall e, d \\
& \sum_{r=1}^{R} Q 3_{y d r}=\sum_{u=1}^{U}\left(\alpha 2_{y u} \times \sum_{k=1}^{K} Q_{u k d}\right), \quad \forall y, d \\
& \sum_{l=1}^{L} Q 4_{t d l}=\sum_{u=1}^{U}\left(\alpha 3_{t u} \times \sum_{k=1}^{K} Q_{u c d}\right), \quad \forall t, d \\
& \sum_{d=1}^{D} Q 5_{e d m}=\beta_{e} \times \sum_{s=1}^{S} Q_{e m s}, \quad \forall e, m \\
& \sum_{p=1}^{P} Q 6_{y r p}=\beta_{y} \times \sum_{d=1}^{D} Q_{y d r}, \quad \forall y, r
\end{aligned}
$$

$$
\begin{aligned}
& \sum_{d=1}^{D} Q_{e d m} \leq F_{m} \times C E_{e m}, \forall e, m \\
& \sum_{d=1}^{D} Q_{y d r} \leq F_{r} \times C Y_{y r}, \forall y, r \\
& \sum_{d=1}^{D} Q_{t d l} \leq C T_{t l}, \forall t, l \\
& \sum_{m=1}^{M} Q 5_{e m s} \leq F m_{m} \times N e_{e s}, \forall e, s \\
& \sum_{r=1}^{R} Q 6_{y r p} \leq F r_{r} \times N y_{y p}, \forall y, p \\
& F d_{d}, F m_{m}, F r_{r}: \text { binary }
\end{aligned}
$$




$$
\sum_{k=1}^{K} Q 1_{u k d} \leq F d_{d} \times C u_{u d}, \forall u, d
$$

$$
\begin{aligned}
& Q 1_{u k d}, Q 2_{e d m}, Q 3_{y d r}, Q 4_{t d l}, \\
& Q 5_{\text {ems }}, Q 6_{y r p} \geq 0
\end{aligned}
$$

\section{Numerical Example}

To illustrate the usefulness of the proposed model, a numerical example is examined in this section. It is assumed that two different types of used products are investigated. In addition, there are two pickup sites (K1, K2), three candidate locations for disassembly centers (D1,D2,D3), three candidate locations for remanufacturing centers (M1, M2, M3), two secondary markets (S1, S2), two demand markets (P1,P2) and one disposal site (L1). These parameters in the model are randomly generated and adopted from $(15,16)$. After solving the proposed model, the objective value is equal to $\$ 52371$ (case 1 -with risk cost) while the objective value is $\$ 44159$ (case 2- without risk cost). The result also indicates that the treatment centers (D1, D2, M1, M3, R2, and R3) should be opened in case 1 whereas D1, D2, M1, M2, R2, and R3 should be constructed in case 2. As a consequence, there are some changes in the flow of items of materials in the RSC network (Q1 $1_{\mathrm{ukd}}, \mathrm{Q} 2_{\mathrm{edm}}, \mathrm{Q} 3_{\mathrm{ydr}}$, and $\left.\mathrm{Q} 4_{\mathrm{tdl}}\right)$ in the two cases (as seen in Figures 2-3). For example, as can be seen in the second stage, 250 units of $2^{\text {nd }}$ used product are delivered from D2 to M1 in case 1 while this figure rises to 330 units shipped from D2 to M1 in the case 2. The reason is that with considering risk cost, the risk cost from D1 to M1 is higher than that from D2 to M1, so it is better to increase the amount of used products delivered from D2 to M1 in order to achieve the total cost minimization.

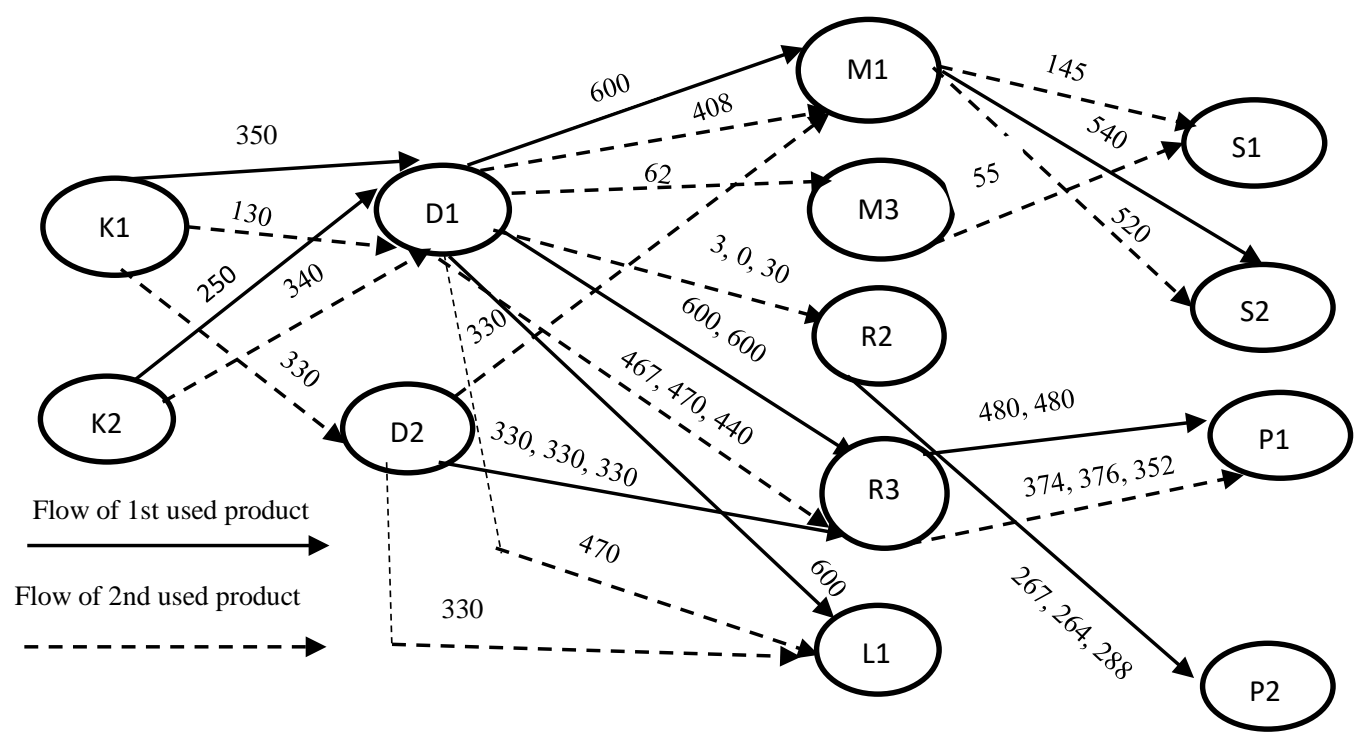

Figure 2. The flow of items and materials with considering risk cost (case 1). 


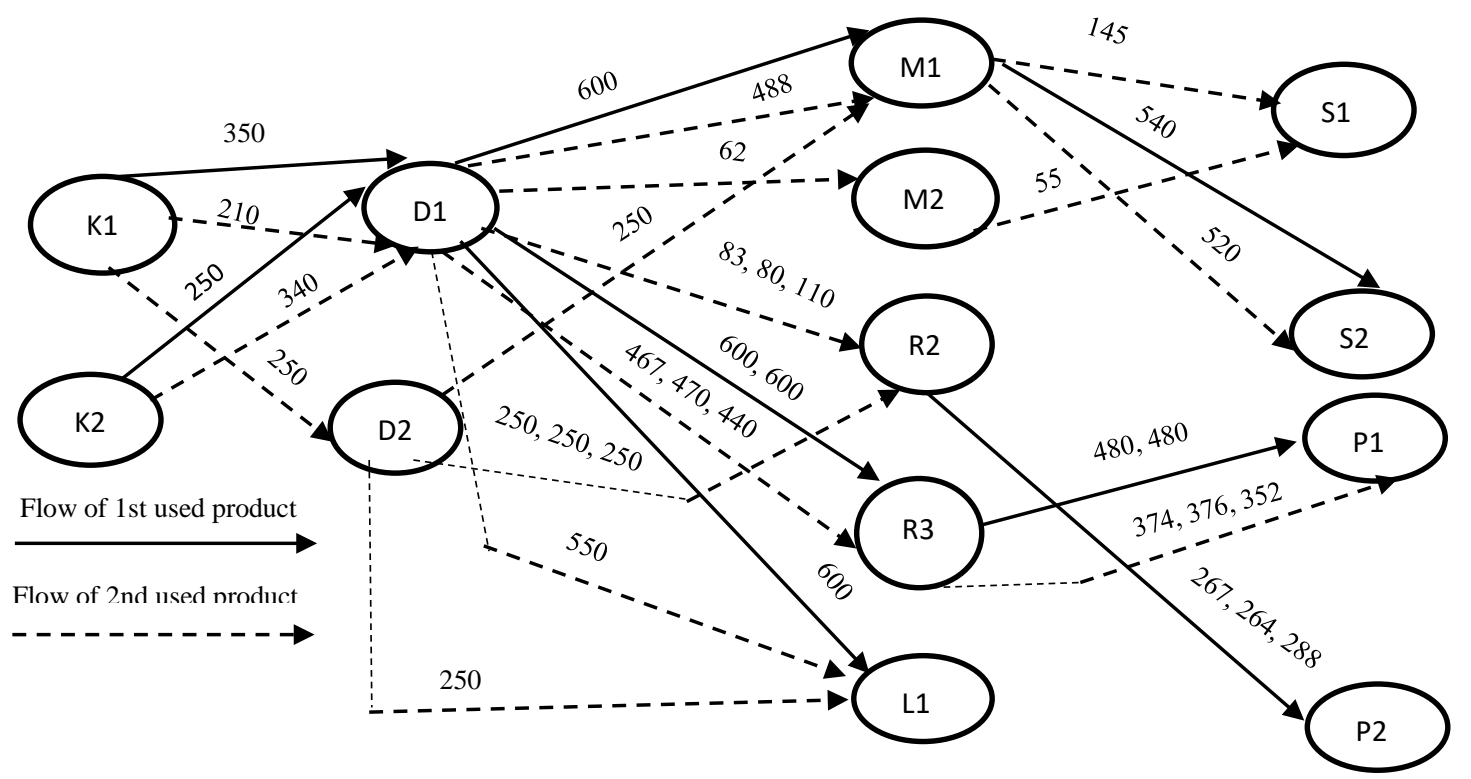

Figure 3. The flow of items and materials without considering risk cost (case 2).

\section{Conclusions}

In this paper, a proposed model for a multi-product and multi-tier RSC has been developed. This model is to optimize the total cost with comprising transportation risk within RSC system. Risks might arise from shipping hazardous materials involved in E-waste, damages due to accidents, delays in pick-up, and ect. From the numerical example, it is clear that the movement of items and materials can be significantly affected with considering risk cost. The proposed model can help managers to have better perception in establishing E-waste RSC system in consideration of risk factors in order to achieve the desired goal.

However, there are some limitations in this research waiting for the further research. Firstly, we assume that the amount of returned products and the cost is deterministic so stochastic or fuzzy approaches should be developed to deal with uncertain elements. Secondly, the proposed model only consider transportation risk, and other risks in the RSC operation such as demand risk, processing risk, or environmental risk which can be incorporated in the future research. In the further development, these limitations are expected to address to have a comprehensive model for E-waste RSC.

The notations and decision variables are presented as below: Indexes:

$k \quad$ Pick-up sites, $k \in\{1 . . K\}$

$d \quad$ Disassembly centers, $d \in\{1 . . D\}$

$m$ Remanufacturing centers, $m \in\{1 . . M\}$

$r \quad$ Recycling centers, $r \in\{1 . . R\}$

$s \quad$ Secondary markets, $s \in\{1 . . S\}$

$p \quad$ Demand markets, $p \in\{1 . . P\}$

Decision variables:

$Q c_{i a b}$

The quality of used product or component $i, i \in\{u, e, y, t\}$ delivered from center $a$, $a \in\{k, d, m, r\}$ to center $b, b \in\{d, m, r, s, p, l\}$, and $c \in\{1, . ., 6\}$

$F d_{d}, F m_{m}, F r_{r}$ Binary variables

Parameters:

$N_{u k} \quad$ The need of used product $u$ at pick-up site $k, u \in\{1 . . U\}, k \in\{1 . . K\}$

$T i_{i} \quad$ The unit transportation cost of used product or component $i, i \in\{u, e, y, t\}$

$B C_{b} \quad$ Fixed cost of center $b, b \in\{d, m, r\}$

$L C_{t l} \quad$ The unit cost for toxic substance $t$ at disposal site $l$

$R i_{i b} \quad$ The unit revenue for component $i, i \in\{e, y\}$ market $b, b \in\{s, p\}$ 
Oi $i_{\text {in }}$ The unit operating cost of used product, component $i, i \in\{u, e, y\}$ at treatment centers $n \in\{d, m, r\}$

$T D_{a b} \quad$ Distance between centers $a, a \in\{k, d, m, r\}$ and $b, b \in\{d, m, r, s, p, l\}$

$\alpha c_{e u} \quad$ The number of units of component or material $i \in\{e, y, t\}$ obtained from used product $u$, and $c \in\{1, . .3\}$

$\beta_{e} \quad$ The average percentage of renewable material e obtained at remanufacturing center

$\beta_{y} \quad$ The average percentage of recycling material $y$ recycled at recycling center

$N i_{i b} \quad$ Maximum need of component or material $i, i \in\{e, y\}$ at market $b, b \in\{s, p\}$

$C i_{i b} \quad$ Maximum capacity for component and material $i, i \in\{u, e, y, t\}$ at center $b, b \in\{d, m, r, l\}$

$P O c_{a b}$ Probability of occurrence of an accident on route $a, a \in\{k, d, m, r\}$ to $b, b \in\{d, m, r, s, p, l\}$, and $c \in\{1, \ldots, 6\}$

$C O c_{a b}$ The consequence of occurrence of an accident on route $a, a \in\{k, d, m, r\}$ to $b, b \in\{d, m, r, s, p, l\}$, and $c \in\{1, \ldots, 6\}$

\section{References}

1. Cucchiella F, D’Adamo I, Lenny Koh SC, Rosa P. Recycling of WEEEs: An economic assessment of present and future e-waste streams. Renew Sustain Energy Rev. 2015 Nov 1;51:263-72.

2. Dowlatshahi S. Developing a Theory of Reverse Logistics. Interfaces (Providence). 2000 Jun 1;30(3):143-55.

3. Govindan K, Soleimani H. A review of reverse logistics and closed-loop supply chains: a Journal of Cleaner Production focus. J Clean Prod. 2017 Jan 20;142:371-84.

4. Demirel E, Demirel N, Gökçen H. A mixed integer linear programming model to optimize reverse logistics activities of end-of-life vehicles in Turkey. J Clean Prod. 2016 Jan 20;112:2101-13.

5. Galvez D, Rakotondranaivo A, Morel L, Camargo M, Fick M. Reverse logistics network design for a biogas plant: An approach based on MILP optimization and Analytical Hierarchical Process (AHP). J Manuf Syst. 2015 Oct 1;37:616-23.

6. Dat LQ, Truc Linh DT, Chou S-Y, Yu VF. Optimizing reverse logistic costs for recycling endof-life electrical and electronic products. Expert Syst Appl. 2012 Jun 1;39(7):6380-7.

7. John ST, Sridharan R, Kumar PNR. Multi-period reverse logistics network design with emission cost. Int J Logist Manag. 2017 Feb 13;28(1):127-49.

8. Fleischmann M, Beullens P, Bloemhof-ruwaard JM, Wassenhove LN van. The impact of product recovery on logistics network design. Prod Oper Manag. 2009 Jan 5;10(2):156-73.

9. John ST, Sridharan R. Modelling and analysis of network design for a reverse supply chain. J Manuf Technol Manag. 2015 Jul 6;26(6):853-67.

10. Kilic HS, Cebeci U, Ayhan MB. Reverse logistics system design for the waste of electrical and electronic equipment (WEEE) in Turkey. Resour Conserv Recycl. 2015 Feb 1;95:120-32.

11. Kumar SK, Tiwari MK, Babiceanu RF. Minimisation of supply chain cost with embedded risk using computational intelligence approaches. Int J Prod Res. 2010 Jul;48(13):3717-39.

12. Thun J-H, Hoenig D. An empirical analysis of supply chain risk management in the German automotive industry. Int J Prod Econ. 2011 May 1;131(1):242-9.

13. Fabiano B, Currò F, Palazzi E, Pastorino R. A framework for risk assessment and decisionmaking strategies in dangerous good transportation. J Hazard Mater. 2002 Jul 1;93(1):1-15.

14. Sheu J-B. A coordinated reverse logistics system for regional management of multi-source hazardous wastes. Comput Oper Res. 2007 May 1;34(5):1442-62.

15. Nguyen P, Phuc K, Yu VF, Chou S-Y. Optimizing the Fuzzy Closed-Loop Supply Chain for Electrical and Electronic Equipments. Int J Fuzzy Syst. 2013;15(1):9-21.

16. El Dabee F, Marian R, Amer Y, El Dabee F, Marian R, Amer Y. A genetic algorithm for a simultaneous optimisation of cost-risk reduction under a just-in-time adaption. J Comput Sci. 2014 Dec 1;10(12):2507-17. 


\section{Acknowledgements}

The first author would like to thank Australian Government for sponsoring her PhD program at the University of South Australia, Australia under Endeavour Award. 